\title{
Análisis jurídico a la iniciativa de Ley para regular las instituciones de tecnología financiera y sus efectos en México
}

\section{Legal analysis of the Law initiative to regulate financial technology institutions and their effects in Mexico}

\author{
RUIZ-MEDRANO, Salvador Francisco*† \& GUEVARA-SANGINÉS, Martha Leticia \\ Universidad de Guanajuato, División de Ciencia Económico Administrativas, Campus Guanajuato, Departamento de \\ Gestión y Dirección de Empresas, Departamento de Estudios Organizacionales.
}

ID $1^{\text {er }}$ Autor: Salvador Francisco, Ruiz-Medrano / ORC ID: 0000-0002-8120-2274, CVU - CONACYT ID: 253989

ID $1^{\text {er }}$ Coautor: Martha Leticia, Guevara-Sanginés / ORC ID: 0000-0001-8441-8110, CVU - CONACYT ID: 15695

DOI: $10.35429 / J L A .2019 .7 .3 .17 .23$

Recibido 26 de Enero, 2019; Aceptado 30 de Marzo, 2019

\section{Resumen}

El pasado 10 de octubre del año 2018, se presentó ante la Cámara de Senadores la iniciativa de decreto para expedir la Ley para Regular las Instituciones de Tecnología Financiera. Esta Ley, tiene como finalidad el de otorgar un soporte de carácter jurídico a los servicios de carácter financiero dentro de las nuevas tecnologías, el análisis de esta Ley se vuelve crucial para entender las nuevas dinámicas de carácter económico y empresarial en nuestro país. Objetivos. El objetivo primario es desentrañara la auténtica ratio legis de esta nueva Ley desde una interpretación literal y apegada al formalismo jurídico. El método empleado para esta disertación se compone de un análisis lógico - jurídico de tipo deductivo para encontrar el auténtico sentido que pretende darle el legislador y sus motivaciones para crear esta reglamentación. Se espera que este análisis contribuya a una mejor comprensión de la Ley y sus contribuciones en el aspecto social y Económico que acarreara en un lapso relativamente corto.

Instituciones de tecnología financiera, e-commerce, crowdfunding, criptomonedas

\begin{abstract}
On October 10, 2018, the decree initiative to issue the Law to Regulate Financial Technology Institutions was presented to the Senate. The purpose of this Law is to grant legal support to financial services within the new technologies, the analysis of this Law becomes crucial to understand the new economic and business dynamics in our country. Goals. The primary objective is to unravel the authentic ratio legis of this new Law from a literal interpretation and attached to legal formalism. The method used for this dissertation is composed of a logical-legal analysis of deductive type to find the true meaning that the legislator intends to give it and its motivations to create this regulation. It is expected that this analysis will contribute to a better understanding of the Law and its contributions in the social and economic aspects that will lead to a relatively short period of time.
\end{abstract}

Financial technology institutions, e-commerce, crowdfunding, cryptocurrencies

Citación: RUIZ-MEDRANO, Salvador Francisco \& GUEVARA-SANGINÉS, Martha Leticia. Análisis jurídico a la iniciativa de Ley para regular las instituciones de tecnología financiera y sus efectos en México. Revista de Aplicaciones del Derecho. 2019. 3-7: 17-23.

\footnotetext{
*Correspondencia al Autor (Correo Electrónico: salvador.rm@ugto.mx)

$\uparrow$ Investigador contribuyendo como primer autor.
} 


\section{Introducción}

En octubre de 2017 se presentó ante el Presidente de la Mesa Directiva de la Cámara de Diputados del Congreso de la Unión, la Iniciativa de Decreto por el que se expide la Ley de Tecnología Financiera y se reforman, adicionan y derogan diversas disposiciones de la Ley de Instituciones de Crédito, la Ley del Mercado de Valores, la Ley General de Organizaciones y Actividades Auxiliares del Crédito, la Ley para la Transparencia y Ordenamiento de los Servicios Financieros, la Ley para Regular las Sociedades de Información Crediticia, la Ley de Protección y Defensa al Usuario de Servicios Financieros, Ley para Regular las Agrupaciones Financieras, la Ley de Firma Electrónica Avanzada, la Ley de la Comisión Nacional Bancaria y de Valores, la Ley Federal para la Prevención e Identificación de Operaciones con Recursos de Procedencia Ilícita y la Ley General de Títulos y Operaciones de Crédito, es decir, y para abreviar en el presente estudio, la Iniciativa Fin Tech, la cual se presenta ante el Senado de la Republica, obteniendo su aprobación el 5 de diciembre de 2017 y que se espera que sea turnada a la Cámara de Diputados y finalmente aprobada hasta el primer trimestre de 2018, señala en su exposición de motivos, que se trata de una suplir una insuficiencia legal acorde al Plan Nacional de Desarrollo 2013-2018 en razón de la necesidad de llevar a cabo una autentica inclusión financiera en nuestro país, ya que se trata de una inclusión financiera a través del empleo de la tecnología y poder llevar estos servicios al mayor número de personas posible, así, esta iniciativa tiene como objetivo el de "...regular las actividades financieras que se realizan a través de la tecnología, en particular a las instituciones de financiamiento colectivo, las instituciones de fondos de pago electrónico, las instituciones de administración de activos virtuales, a las cuales se les denominará en su conjunto para efectos de la Ley como Instituciones de Tecnología Financiera (ITF)." ya que “...la tecnología financiera permite que la población tenga a su alcance nuevos modelos de negocio para atender sus necesidades, llegando a lugares y sectores de la población que actualmente no utilicen el sistema financiero para realizar sus transacciones o que, usándolo, requieran de nuevos medios para llevarlas a cabo." (Presidencia de la República. México., 2017, pág. 3).
La misma iniciativa presenta los principios en los que se sustenta la misma, que son los de inclusión financiera, protección al consumidor, preservación de la estabilidad financiera, promoción de la competencia y prevención de lavado de dinero y financiamiento al terrorismo. Dentro de estos principios, cabe destacar el de preservación de la estabilidad financiera, promoción de la competencia y prevención de lavado de dinero y financiamiento al terrorismo, donde se busca en un primer momento establecer un marco general de autorización y operación supervisada a las ITF y buscando el correcto funcionamiento financiero y su integridad, estableciendo el marco regulatorio ya aceptado y probado, con estándares mínimos de identificación del cliente, en donde además, para poder operar las instituciones requerirán de una autorización ante la Comisión Nacional Bancaria y de Valores, la cual se otorgará previa revisión de la información y documentación que se menciona en dicha ley y previo acuerdo del Comité de Instituciones de Tecnología Financiera. Dicho Comité estaría integrado por las tres autoridades financieras clave, a saber, la Secretaría de Hacienda y Crédito Público, la Comisión Nacional Bancaria y de Valores y el Banco de México. Un elemento clave en la regulación es la obligación de implementar una política de separación de cuentas y el uso de una cuenta de depósito de dinero en una institución de crédito o entidad financiera facultada para recibir depósitos, pretendiendo reducir el riesgo de mal uso de los recursos de los clientes de las Instituciones de Tecnología Financiera.

\section{Las observaciones de la Comisión Federal de Mejora Regulatoria (COFEMER)}

Dentro de la elaboración de la Ley FINTECH, hubo un momento en que se enviaron en septiembre de 2017 a la Comisión Federal de Mejora Regulatoria (COFEMER), el anteproyecto de la misma para la elaboración de un Dictamen Total, el cual se envió con fecha de 28 septiembre de 2017 al Dr. Miguel Messmacher Linartas, Subsecretario de Ingresos de la Secretaria de Hacienda y Crédito Público, firmado por Julio César Rocha López, Coordinador General de este organismo, en dónde pone manifiesto una serie de cuestiones en torno a esta ley. 
De esta forma, en el aspecto de las consideraciones, se menciona que la aparición de estos sistemas de carácter financiero apoyados en las tecnologías obedecen a una crisis de confianza de parte de los usuarios y consumidores derivado de las crisis subprime de 2008 lo que ocasiono que los emprendedores empezaran a ofrecer alternativas en este sentido, delegando un grado de participación prioritario a las personas a través de las plataformas portátiles e internet, en dónde cabria mencionar que estos productos logran más éxito en la medida en que sean transparentes, prácticos, de fácil acceso y cercanos a los clientes (Chishti \& Barberis, 2016), en dónde además ha de destacarse el hecho de que derivado de la facilidad, abaratamiento, y difusión del uso de las nuevas tecnologías y del empleo del Internet, se han incrementado las operaciones de comercio electrónico en dónde el país ya cuenta con "180 empresas con tecnología financiera, donde la actividad de dichas empresas se concentra en cuatro segmentos principales: i) plataformas de finanzas alternativas con 59 emprendimientos: ii)pagos con 50; iii) gestión de finanzas 2ersonales con 22, y iv) finanzas empresariales con 21 .

Tales segmentos representan el $84.4 \%$ de las empresas FinTech en el país." (Comision Federal de Mejora Regulatoria, 2017, pág. 6) por lo que resulta indispensable contar con un documento legal que proporcione certeza jurídica en cuanto a las operaciones que realizan estas empresas.
Este mismo dictamen, la COFEMER señala los puntos clave que pretende regular la Ley FINTECH, que son el uso de instituciones tecnológicas, el uso de plataformas de financiamiento colectivo o crowdfunding, regulación de activos virtuales y las sociedades autorizadas para operar con Modelos Novedosos, en donde igualmente se señalan algunos antecedentes regulatorios del ámbito internacional sobre estos temas (Comision Federal de Mejora Regulatoria, 2017, págs. 910), para finalmente concluir en un análisis costo-beneficio que tendrá esta Ley, los cuales fueron evaluados en torno de los costos que tendrá la creación de estos modelos de negocios versus los beneficios de la población al acceder a estos esquemas aunado a las ventajas que otorgaría la certeza jurídica de contar con una ley de esta naturaleza, y un análisis de impacto en la competencia, la cual, señala que "estimula la eficiencia del mercado dado que filtran los oferentes en razón del cumplimiento de los requisitos mínimos que debe contar la persona moral que quiera operar bajo alguna de las diferentes figuras previstas en la ley" (Comision Federal de Mejora Regulatoria, 2017, pág. 24)

\section{Análisis general de la iniciativa de la Ley}

Desde el inicio de la iniciativa se manifiesta que es de orden público y observancia general en todo el territorio nacional, cuyo objetivo es la de regular los servicios financieros que prestan las instituciones de tecnología financiera y cuya base son los principios de inclusión e innovación financiera, promoción de la competencia y protección al consumidor, siendo la Comisión Nacional Bancaria y de Valores y al Banco de México la supervisión del cumplimiento de esta Ley, y estableciendo desde un comienzo el plazo, que no podrá exceder de 90 días, en que estas autoridades deberán pronunciarse sobre el deseo de una persona física o moral sobre la autorización de operar como una Institución de Tecnología Financiera o ITF como se menciona en la Ley, quienes serán las depositarias de la responsabilidad ante las autoridades y ante las personas y quienes podrán operar de tres formas diferentes (Presidencia de la República. México., 2017, págs. 10-17). 


\section{Las operaciones de las ITF}

Las ITF para poder operar deben de contar con una autorización de la Comisión Nacional Bancaria y de Valores (CNBV), las cuales deberán de evitar la difusión de información falsa o engañosa y deberán de difundir información que permita a los clientes identificar los riesgos de las operaciones que realicen, dejando en claro que, tanto el Gobierno Federal ni las entidades de la administración pública paraestatal podrán responsabilizarse o garantizar los recursos de los clientes que utilicen los servicios de las ITF, ni podrán responsabilizarse de las operaciones de las ITF o clientes con otras ITF, poniendo en claro que dicho aviso o advertencia deberá de darse a conocer a través de cualquier medio, tanto electrónico como virtual así como también en los contratos que celebren con los clientes.

Igualmente, las ITF pueden ser de tres tipos, las Instituciones de Tecnología Financiera, Institución de Financiamiento Colectivo e Institución de Fondos de Pago Electrónico.

\section{Las instituciones de Financiamiento Colectivo}

Se definen como ITF de Financiamiento Colectivo a aquellas sociedades que ponen en contacto a personas del público en general para que entre ellas se otorguen financiamientos mediante operaciones de financiamiento de deuda, de financiamiento de capital y financiamiento de copropiedad o regalías. Estas operaciones podrán llevarse a cabo a través de aplicaciones, páginas web o cualquier otro medio de comunicación electrónica o digital, siendo las partes intervinientes en estas operaciones llamadas inversionistas y solicitantes, de acuerdo con lo que pretendan obtener a través de estas operaciones.
Para efectos de la Ley, se considera que el financiamiento colectivo de deuda opera de forma en que los inversionistas otorguen prestamos, créditos, mutuos o cualquier otro financiamiento causante de un pasivo directo al o los solicitantes; el financiamiento colectivo de capital opera con el fin de que los inversionistas compren o adquieran títulos representativos de capital social de personas morales que actúen como solicitantes, y; el financiamiento colectivo de copropiedad o regalías tiene como fin el de que los inversionistas y solicitantes celebren entre ellos asociaciones en participación donde el inversionista adquiera una parte alícuota o participación en un bien presente o futuro que se genere con las actividades o proyectos del solicitante.

Todas estas operaciones se realizaran en moneda nacional y las instituciones de financiamiento colectivo podrán actuar como mandatarias o comisionistas de sus clientes para la realización de las actividades antes reseñadas $\mathrm{y}$ entre sus principales obligaciones, estas deberán de dar a conocer a los posibles inversionistas de forma clara e indubitable los criterios de selección de los solicitantes, los riesgos inherentes a las inversiones que realicen, toda información de comportamiento de los solicitantes, contar con los medios necesarios para lograr la formalización de las operaciones y ser usuarias de una sociedad de información crediticia, sin embargo, estas instituciones tienen prohibido asegurar retornos o rendimientos sobre inversión o garantizar el resultado o éxito de las mismas a los inversionistas.

\section{Las Instituciones de Fondos de Pago Electrónico}

Se les llamara como tales a aquellas instituciones que presten servicios al público de manera habitual y profesional de emisión, administración, redención y transmisión de fondos de pago electrónico a través de aplicaciones, páginas de internet o cualquier otro medio de comunicación electrónica o digital y que cuenten con la autorización de la CNBV. 
Entre sus principales atribuciones están las de abrir o llevar una o más cuentas de fondos de pago electrónico por cliente, realizar trasferencias de fondos de pago electrónico entre sus clientes, realizar trasferencias mediante cargos o abonos en las cuentas de los clientes, entregar las cantidades de dinero o activos virtuales en la cuenta de fondos de pago $\mathrm{u}$ otros usuarios de entidades financieras mediante el respectivo cargo a la cuenta del cliente y mantener actualizado el registro de cuentas de sus clientes.

Igualmente, la Ley les permite emitir, comercializar o administrar instrumentos para la disposición de fondos electrónicos, prestar el servicio de transmisión de dinero, otorgar créditos o prestamos, realizar operaciones con activos virtuales, obtener préstamos o créditos, emitir valores por cuenta propia $y$, en términos amplios todos los actos necesarios para consecución de su fin social.

Entre las obligaciones que la Ley establece se cuentan los de que no podrán concederse con cargo a los fondos o activos virtuales recibidos créditos o prestamos ni podrán cobrar intereses por dichos créditos, los cuales deberán de ser abonado por el cliente cuando reciba de éste fondos o activos virtuales. Asimismo, no podrán pagar a sus clientes intereses ni cualquier otro rendimiento o beneficio monetario por el saldo acumulado de sus clientes.

\section{Las operaciones con Activos Virtuales}

Para efectos de esta Ley, un activo virtual es la representación de valor registrada electrónicamente y utilizada entre el público como medio de pago para todo tipo de actos jurídicos y cuya transferencia únicamente puede llevarse a cabo por medio electrónicos. En ningún caso y bajo ningún concepto el activo virtual puede ser interpretado como moneda de curso legal o en divisas.

Las ITF que operen este tipo de activos solo podrán usar aquellos que sean determinados por el Banco de México, para lo cual requerirán con autorización de esta misma institución.
Igualmente las ITF que operen este tipo de activos, deberán de estar en posibilidades de entregar a sus clientes la cantidad de activos virtuales que les soliciten así como el pago en moneda nacional en caso de que el cliente venda sus activos virtuales a las ITF, y será el Banco de México que determine las características de los activos virtuales autorizados para las operaciones de estas ITF y señalara las medidas a que deberán sujetarse para la custodia y control sobre sus activos virtuales que posean, es decir la posesión de firmas, claves o autorizaciones que sean suficientes para ejecutar sus operaciones.

Estas ITF tienen prohibido de manera taxativa vender, ceder o transferir su propiedad, dar préstamo o garantía o afectar el uso, goce o disfrute de los activos virtuales que custodien y controlen por cuenta de sus clientes y deberán de divulgar a sus clientes los riesgos que existen por celebrar operaciones con dichos activos y deberán de informales, como mínimo lo siguiente:

i. El activo virtual no es moneda de curso legal y no está respaldada por el Gobierno Federal, ni por el Banco de México.

ii. La imposibilidad de revertir las operaciones una vez ejecutadas, en su caso.

iii. La volatilidad del activo virtual.

iv. Los riesgos tecnológicos, cibernéticos y de fraude inherentes a los activos virtuales.

\section{El derecho como fuente de las monedas virtuales}

La importancia de este apartado subyace en la necesidad de encontrar una fuente del derecho que se adapte a un concepto esquivo como lo son las monedas virtuales, que esconde un halo de duda en su exigibilidad, puesto que si no se le encuentra un fundamento legal pierde toda validez y pasa a ser una laguna, en el mejor de los casos o bien una causa de nulidad de instrumentos jurídicos que invocan a dicho dinero virtual como fuente de una obligación. 


\section{De los derechos reales y de los personales}

El derecho real es una relación de derecho por virtud de la cual una persona tiene la facultad de obtener de una cosa, exclusivamente, y en una forma oponible a todos, toda la utilidad que produce o parte de ella. Por lo anterior, el de facultar la propia conducta de un sujeto, sobre una cosa determinada, con las limitaciones que imponga la naturaleza de la cosa, la ley, así como los derechos de terceros que afecten su ejercicio, y que da derecho a aprovechar esa cosa en diferentes grados según la naturaleza del derecho real de que se trate; sin embargo, el poder jurídico que se tiene sobre la cosa no comprende necesariamente su utilización material, porque es suficiente que el titular del derecho real reciba de ella un beneficio de cualquier orden.

Asimismo, en los derechos reales debe existir la cosa objeto del derecho real, mientras que en los derechos personales se admite que su objeto no exista al constituirse la obligación o el contrato, como en la venta de una cosa futura. De lo aquí señalado, por tanto, se ha de concluir que las monedas virtuales no encuadran en la definición dada anteriormente puesto que el derecho no cae en una cosa, puesto que es a futuro, intangible y no existe en el momento.

Por otro lado, el derecho personal o de crédito, denominado también obligación, es una relación de derecho en virtud de la cual una persona, el acreedor, tiene el derecho a exigir de otra, el deudor, el cumplimiento de una prestación determinada, positiva o negativa. Esta relación jurídica se llama crédito desde el punto de vista activo y deuda desde el pasivo. El derecho de crédito se encuentra protegido por acciones personales, dirigidas en contra del deudor, de sus fiadores o de sus herederos; es decir, en el derecho personal, quien se encuentra obligado a cumplir con la prestación de dar, hacer o no hacer es un sujeto individualmente determinado, llamado sujeto pasivo o deudor. La definición del término persona implica en general un grado importante de complejidad en virtud de las diversas acepciones que conlleva.
Ahora bien, es en esta categoría donde puede ser encuadrado el dinero virtual puesto que en los derechos personales la prestación consiste en dar, hacer o no hacer, con la diferencia con respecto a los reales, de que, en el derecho personal, del comportamiento del sujeto pasivo depende que la obligación se cumpla, es decir puede ser a futuro. Así, el derecho de crédito, de carácter personal, el objeto es una prestación del deudor, si bien es cierto que este tipo de derechos solamente pueden hacerse efectivos contra personas determinadas, vinculadas por una relación jurídica, entiéndase un contrato; igualmente se ha de considerar que los derechos personales son ilimitados e infinitos, pues las partes pueden crear todos los que convengan a sus intereses, sujetándose al contrato como fuente de la obligación.

Sin embargo hay que preguntarse ¿Dónde nace la obligación de aceptar algo que en origen no existe, es intangible y solo existirá al momento del cumplimiento de la obligación?, la respuesta es la costumbre, la cual es fuente del derecho, que se le suele definir como la obra inconsciente o subconsciente de la masa, cuyo elemento subjetivo alude a un estado psicológico consciente de la colectividad (Mauricio Figueroa, 2004, pág. 64 y ss.), es decir, sin el ánimo de entrar en el campo de la metafísica, se trata de que la fuerza de sus existencia radica en la creencia que tenemos de manera colectiva de que existe y es válido exigirlo ante la autoridad y es en este punto dónde la iniciativa del ejecutivo otorga certeza a aquellas personas que decidan contratarse con entidades que trafiquen en el comercio con este tipo de monedas de carácter virtual.

\section{De las autorizaciones para operar como ITF}

Las personas que pretendan realizar las actividades antes mencionadas deberán de solicitar su autorización como ITF ante la CNBV, quien la otorgara discrecionalmente previo acuerdo del Comité Interinstitucional, quien estará integrado por seis miembros propietarios de la Secretaria de Hacienda y Crédito Público, Banco de México y de la propia CNBV quienes revisaran la información de los solicitantes. 
Los solicitantes deberán de ser sociedades anónimas y que en sus estatutos se señale que su objeto social este acorde con las actividades señaladas en la presente Ley, prometan sujetarse a la misma, que su domicilio se encuentre en territorio nacional, contar con el capital mínimo para llevar a cabo sus actividades. Igualmente deberán de contar con un plan de negocios, las políticas de separación de cuentas, las políticas de divulgación de riesgos y responsabilidades por la realización de sus actividades como ITF, las medidas y políticas en materia de control de riesgos operativos y seguridad de la información, incluyendo las políticas de confidencialidad. Igualmente deberán de contar con los procesos operativos y de control de identificación de sus clientes, las políticas de solución de controversias durante la realización de sus actividades, las políticas de prevención de fraudes y prevención de operaciones con recursos de procedencia ilícita y financiamiento al terrorismo.

Deberán de incluir también toda información relativa a sus contratos con otras ITF o proveedores de servicios tecnológicos necesarios para la realización de sus actividades, la información de las personas que directa o indirectamente mantengan o pretendan mantener una participación en el capital social de la ITF, información relativa al administrador o consejeros de la ITF, la información necesaria para comprobar que son titulares o tienen derecho al uso de la interfaz, página de internet o medio de comunicación electrónico o digital. Toda esta información deberá de ser verificada por la CNBV.

\section{Conclusiones}

En el ámbito legal internacional se ha de considerar que esta iniciativa y futura Ley, es de las pocas que existen en el mundo y que presenta los tres grandes aspectos que abarcan las cuestiones relativas al financiamiento tecnológico que son el crowdfunding o financiamiento colectivo, pago electrónico el manejo de activos virtuales, mejor conocidos como cripto monedas.
$\mathrm{Si}$ bien la iniciativa representa un esfuerzo por regular estas nuevas formas de financiamiento y operaciones financieras, no hay que perder de vista que toda la responsabilidad relativa a las operaciones el Estado mexicano la traslada al individuo, sin considerar aspectos como la ignorancia, mala fe o dolo en que pudieran operar estas ITF, lo que deja en relativo estado de indefensión al individuo, puesto que basta con recordar que el pueblo mexicano no está educado desde el aspecto de las finanzas y el uso de las nuevas tecnologías en el plano financiero. Igualmente, no hay que olvidar que ya en otros Estados del planeta, como es el caso de España, las monedas virtuales, su novedad y moda sirvieron de fachada para la creación de esquemas de fraude piramidal como lo son el caso "Únete" o el caso "Scoin", que incluso este último sirvió de lavado de dinero en la isla de Malta, estos fraudes pueden repetirse en el caso de México dejando a la gente con escasas armas legales ante los mismos. Ahora bien, no hay que perder de vista que esta iniciativa puede ser perfectible a lo largo de su recorrido, por lo que aún pueden existir mejoras en este sentido, y a grosso modo es un esfuerzo loable por tratar de contar con certeza jurídica para este tipo de operaciones.

\section{Referencias}

Comision Federal de Mejora Regulatoria. (29 de septiembre de 2017). COFEMER SIMIR SE. Recuperado el 7 de enero de 2018, de MANIFESTACIÓN DE IMPACTO REGULATORIO (SIMIR) Dictamen Final Total COFEME/17/5800: http://www.cofemersimir.gob.mx/expediente/20 718/emitido/46468/COFEME_17_5800

Chishti, S., \& Barberis, J. (2016). The FINTECH Book: The Financial Technology Handbook for Investors, Entrepreneurs and Visionaries. Hoboken, New Jersey: Wiley.

Mauricio Figueroa, L. (2004). Las fuentes del derecho. México D.F.: Porrúa.

Presidencia de la República. México. (10 de octubre de 2017). Senado de la República. Recuperado el 18 de diciembre de 2017, de Gaceta parlamentaria: http://infosen.senado.gob.mx/sgsp/gaceta/63/3/ 2017-10-12-

1/assets/documentos/Iniciativa_Ejecitvo_Feder al.pdf 